\title{
Comparison of usefulness of clinical diagnostic criteria for hepatocellular carcinoma in a hepatitis B endemic area
}

\author{
So Young Bae, Moon Seok Choi, Geum-Youn Gwak, Yong Han Paik, Joon Hyoek Lee, Kwang Cheol Koh, \\ Seung Woon Paik, and Byung Chul Yoo \\ Department of Internal Medicine, Samsung Medical Center, Sungkyunkwan University School of Medicine, Seoul, Korea
}

Background/Aims: We compared the accuracy and usefulness of clinical diagnostic criteria for hepatocellular carcinoma in a hepatitis B virus (HBV)-endemic area.

Methods: We reviewed the medical records of 355 patients who had undergone liver resection or biopsy at our institution between January 2008 and December 2009. These patients were reevaluated using four noninvasive diagnostic criteria for hepatocellular carcinoma proposed by the European Association for the Study of the Liver (EASL), the American Association for the Study of Liver Diseases (AASLD), the Korean Liver Cancer Study Group and the National Cancer Center (KLCSG/NCC), and National Comprehensive Cancer Network (NCCN) guidelines.

Results: The overall sensitivity was highest using the KLCSG/NCC criteria (79.8\%), followed by the AASLD (51.5\%), EASL $(38.4 \%)$, and NCCN $(10.1 \% ; P<0.001)$ criteria, whereas the specificity $(84.5-98.3 \%)$ and positive predictive value $(96.2-$ 98.3\%) were similar for all of the criteria. The KLCSG/NCC criteria had an acceptable false-positive rate and the highest sensitivity among all of the patients, including those positive for $\mathrm{HBsAg}$, those without liver cancer, and those with a tumor of at least $2 \mathrm{~cm}$.

Conclusions: The KLCSG/NCC and AASLD criteria exhibited the highest sensitivity, and all four guidelines had a high specificity among all of the patients. Based on the sensitivity and false-positive rate, the KLCSG/NCC criteria was the most useful in the majority of patients. Inclusion of HBV infection in the clinical diagnostic criteria for hepatocellular carcinoma would be reasonable and may lead to an improvement in the sensitivity, with acceptable false-positive rates, in HBVendemic areas. (Clin Mol Hepatol 2012;18:185-194)

Keywords: Comparison; Clinical diagnostic criteria; Hepatocellular carcinoma

See Editorial on Page 174

\section{INTRODUCTION}

Hepatocellular carcinoma (HCC) is the fifth most common can- cer and the third leading cause of cancer mortality in the world. ${ }^{1}$ HCC mainly occurs in patients with high risk factors including hepatitis B virus (HBV) infection, hepatitis C virus (HCV) infection, and liver cirrhosis $(\mathrm{LC}){ }^{2}$ In Korea, where about $5-6 \%$ of general population has HBV infection, HCC is one of top three cancers, imposing a significant health problem nationwide. ${ }^{3}$

\footnotetext{
Abbreviations:

AASLD, the American Association for the Study of Liver Diseases; AFP, alpha fetoprotein; CC, cholangiocarcinoma; CT, computed tomography; EASL, European Association for the Study of the Liver; HBV, hepatitis $B$ virus; $\mathrm{HCC}$, hepatocellular carcinoma; $\mathrm{HCV}$, hepatitis C virus; KLCSG/ NCC, the Korean Liver Cancer Study Group and the National Cancer Center; LC, liver cirrhosis; MRI, magnetic resonance imaging; NCCN, National Comprehensive Cancer Network; PPV, positive predictive value
}

\section{Corresponding author : Moon Seok Choi}

Department of Internal Medicine, Samsung Medical Center,

Sungkyunkwan University School of Medicine, 81 Irwon-ro,

Gangnam-gu, Seoul 135-710, Korea

Tel. +82-2-3410-3409, Fax. +82-2-3410-6983, E-mail; drmschoi@gmail.com

Received : December 23, 2011

Revised: April 9, 2012

Accepted : April 9, 2012

Copyright $\odot 2012$ by The Korean Association for the Study of the Liver

This is an Open Access article distributed under the terms of the Creative Commons Attribution Non-Commercial License (http://creativecommons.org/licenses/by-nc/3.0/) which permits unrestricted non-commercial use, distribution, and reproduction in any medium, provided the original work is properly cited. 
Elevated serum alpha-fetoprotein (AFP) and typical enhancement pattern during dynamic imaging provide critical clues for the diagnosis of HCC. ${ }^{4,5}$ Although sensitivity and specificity of serum AFP as a tumor marker is being challenged, high level or steadyincreasing level of serum AFP strongly suggest development of HCC. ${ }^{6}$ Arterial hyperattenuation and washout in the portal or delayed phase at contrast-enhanced computed tomography (CT) or dynamic magnetic resonance imaging (MRI) have been accepted as typical enhancement patterns of $\mathrm{HCC}^{7.8}$

Clinical diagnosis of HCC without biopsy is a routine clinical practice, ${ }^{9}$ since guidelines by the European Association for the Study of the Liver (EASL) ${ }^{10}$ and the American Association for the Study of Liver Diseases (AASLD) ${ }^{11}$ have proposed clinical diagnosis of HCC based on a typical enhancement pattern during dynamic imaging and/or elevated serum AFP level in high-risk groups. Usefulness of the clinical diagnostic criteria by Western guidelines has not been fully evaluated in HBV endemic area like Korea except one latest report about validation of AASLD guideline in HBV endemic area. ${ }^{12}$ In addition to EASL and AASLD guidelines, the National Comprehensive Cancer Network (NCCN) guidelines ${ }^{13}$ and the Korean Liver Cancer Study Group and the National Cancer Center (KLCSG/NCC) guideline ${ }^{14}$ are also used for management of HCC in Korea. These four clinical diagnostic criteria differ in details of risk factors, cut-off value of serum AFP level, and definition of typical image pattern according to their population of interest. However, there is little data comparing the accuracy of various non-invasive diagnostic criteria for HCC.

Herein, we compared the accuracy and usefulness of these clinical diagnostic criteria in a HBV endemic area.

\section{SUBJECTS AND METHODS}

\section{Subjects}

Patient who had undergone liver resection or biopsy at Samsung Medical Center, Seoul, Korea, from January 2008 to December 2009, and who had fulfilled all required examinations for clinical diagnosis of HCC including viral markers, serum AFP, and liver imaging (dynamic CT, gadoxetic acid-enhanced liver MRI, or both) were included in this study and reviewed retrospectively. Patients who had recurrent tumors or multiple tumors were excluded.

\section{Clinical diagnostic criteria}

A total of 355 patients were enrolled in this study. We reviewed their clinical history, risk factors including HBV infection, HCV infection, and LC, serum AFP level, tumor size, enhancement patterns during dynamic imaging modalities, and final histologic diagnosis. LC was diagnosed based on the histologic findings from liver resection or biopsy. It was evaluated whether patients met the clinical diagnostic criteria by EASL, ${ }^{10}$ AASLD $_{1}{ }^{11} \mathrm{NCCN}^{13}$ and KLCSG/NCC guidelines. ${ }^{14}$ Difference in criteria for non-invasive diagnosis among the four guidelines was summarized in Table 1.

\section{Dynamic imaging and liver biopsy}

Triple-phase helical CT examinations were performed using multidetector CT scanners (Lightspeed QX/I, Lightspeed Ultra 8, Lightspeed VCT; GE Healthcare, Buckinghamshire, UK) at $5 \mathrm{~mm}$ slice thickness and $2.5 \mathrm{~mm}$ interval. Images were obtained in the

Table 1. Summary of the four types of clinical diagnostic criteria

\begin{tabular}{|c|c|c|c|}
\hline & Risk group & Size & Serum AFP level and typical image findings \\
\hline \multirow[t]{2}{*}{ EASL } & LC & $\geq 2 \mathrm{~cm}$ & Typical findings on single image if AFP $\geq 400 \mathrm{ng} / \mathrm{mL}$ \\
\hline & & & Typical findings on two images if AFP $<400 \mathrm{ng} / \mathrm{mL}$ \\
\hline \multirow[t]{2}{*}{ AASLD } & LC & $1-2 \mathrm{~cm}$ & Typical findings on two images \\
\hline & & $\geq 2 \mathrm{~cm}$ & Typical findings on single image or AFP $\geq 200 \mathrm{ng} / \mathrm{mL}$ \\
\hline \multirow[t]{3}{*}{ KLCSG/NCC } & HBV, HCV, LC & $<2 \mathrm{~cm}$ & Typical findings on single image if AFP $\geq 200 \mathrm{ng} / \mathrm{mL}$ \\
\hline & & & Typical findings on two images if AFP $<200 \mathrm{ng} / \mathrm{mL}$ \\
\hline & & $\geq 2 \mathrm{~cm}$ & Typical findings on single image \\
\hline \multirow[t]{2}{*}{ NCCN } & & & AFP $\geq 4,000 \mathrm{ng} / \mathrm{mL}$ if $\mathrm{HBV}(+)$ \\
\hline & & & AFP $\geq 400 \mathrm{ng} / \mathrm{mL}$ if HBV (-) \\
\hline
\end{tabular}

EASL, European Association for the Study of the Liver; AASLD, the American Association for the Study of Liver Diseases; KLCSG/NCC, the Korean Liver Cancer Study Group and the National Cancer Center; NCCN, National Comprehensive Cancer Network; LC, liver cirrhosis; HBV, hepatitis B virus; HCV, hepatitis C virus; AFP, alpha fetoprotein. 
craniocaudal direction. Hepatic arterial phase scanning began 30-35 seconds after injection of $120 \mathrm{~mL}$ of a nonionic iodinated contrast material (iopamidol, Iopamiro 300; Bracco, Milan, Italy) at a rate of 3-4 mL/s using a bolus triggered technique. The portal and equilibrium phases of scanning began 70 seconds and 180 seconds after injection of the contrast material, respectively. ${ }^{15}$

MRI was performed with a $1.5 \mathrm{~T}$ and $3.0 \mathrm{~T}$ whole-body MRI (Intera Achieva; Philips Medical Systems, Bothell, Netherlands). Gadoxetic acid disodium (Primovist ${ }^{\circledR}$; Bayer Schering Pharma, Berlin, Germany) were used as superparamagnetic iron oxide agents. The dose of gadoxetic acid disodium was infused $(0.1 \mathrm{~mL} / \mathrm{kg})$ in patients. The contrast agent was manually administered intravenously through an in-line 5 - $\mu \mathrm{m}$ specific filter with a rapid bolus in 1 second, immediately followed by a $10-\mathrm{mL}$ saline solution flush. The entire procedure was performed in approximately 5 seconds. MRI was initiated 10 minutes after the injection of the contrast agent. $^{15,16}$

Percutaneous biopsy of nodule in the liver was done under the guidance of real-time sonography with the freehand technique. The tru-cut biopsy needles (Gunbiopsy Needle, M.I.Tech \& ACECUT biopsy needle, TSK) were used. These needles are springloaded and fully automated. The outer diameters of the tru-cut needles were 18-gauge. ${ }^{16,17}$ Specimens of percutaneous liver biopsy and surgical specimens were reviewed by experienced hepatic pathologists.

\section{Statistical analysis}

Accuracy of the non-invasive criteria including sensitivity, specificity, and positive predictive value (PPV) was compared among the four guidelines in 355 included patients. In addition, accuracy of each diagnostic criteria was analyzed according to presence of HBV infection, accompanying LC, and tumor size. Finally, sensitivity and specificity of the diagnostic criteria was compared in $\mathrm{HBSAg}$ $(+)$ or (-) subgroups with or without LC. EASL criteria were not applicable in those without LC. In addition, EASL and AASLD criteria were not applicable in patients with tumor $<2 \mathrm{~cm}$, either. Hence, accuracy of the criteria was not assessed in those subgroups. Finally, usefulness of each clinical diagnostic criteria was compared based on sensitivity and false positive rate. Sensitivity represents the proportion of patients with HCC in whom invasive diagnosis can be avoided with application of clinical diagnostic criteria. False positive rate (FPR) indicates the proportion of patients who was misdiagnosed as HCC with application of clinical diagnostic criteria. FPR $<5 \%$ was considered to be acceptable. Since all these four clinical criteria recommend biopsy or close follow up for suspicious lesions which do not meet the criteria, specificity is not so critical concern in assessment of their usefulness.

We used the Generalized Estimating Equations model for comparing sensitivity, specificity, and PPV of the four guidelines. Fisher's exact test with Bonferroni's correction was performed for analysis of accuracy of each criteria according to HBV infection status, presence of $L C$, and tumor size. These statistical analyses were performed using PASW version 18.0 (SPSS, Chicago, IL, USA.) and R version 2.11.1. A $P$-value $<0.05$ was considered as significant.

\section{RESULTS}

\section{Baseline characteristics of patients}

The median age of the 355 patients was 54.7 (range 18-80) years and the gender ratio (M:F) was 3.3:1 (Table 2). Of these, 253 patients (71.3\%) had HBsAg, 19 (5.4\%) had HCV infection. One hundred and eighty patients (50.7\%) showed LC. Serum AFP

Table 2. Baseline characteristics of 355 patients

\begin{tabular}{lc}
\hline Number of patients (\%) & \\
\hline Age (yr, median) & 272.7 (range, 18-80) \\
Gender (M:F) & \\
\hline Risk factors & $253(7.3: 1)$ \\
\hline HBV & $19(5.4 \%)$ \\
\hline HCV & $180(50.7 \%)$ \\
\hline LC & \\
\hline Serum AFP (ng/mL) & $274(77.1 \%)$ \\
\hline$<200$ & $19(5.4 \%)$ \\
\hline $200-400$ & $62(17.5 \%)$ \\
\hline$>400$ & \\
\hline Size of tumor & $62(17.5 \%)$ \\
\hline$<2$ cm & $293(82.5 \%)$ \\
\hline$\geq 2$ cm & $264(74.4 \%)$ \\
\hline Method of histologic diagnosis & $4(1.1 \%)$ \\
\hline Hepatic resection & $72(20.3 \%)$ \\
\hline Liver transplantation & $15(4.2 \%)$ \\
\hline Liver biopsy & \\
\hline Hepatic resection + liver biopsy & \\
\hline
\end{tabular}

HBV, hepatitis B virus; HCV, hepatitis C virus; LC, liver cirrhosis; AFP, alpha fetoprotein. 
Table 3. Final histologic diagnosis of the patients

\begin{tabular}{lc}
\hline Final histologic diagnosis & \\
\hline HCC & $284(80.0 \%)$ \\
CC & $23(6.5 \%)$ \\
\hline HCC-CC & $13(3.7 \%)$ \\
\hline Focal nodular hyperplasia & $10(2.8 \%)$ \\
Eosinophilic abscess & $5(1.4 \%)$ \\
Abscess & $3(0.8 \%)$ \\
Regenerating nodule & $2(0.6 \%)$ \\
Others & $15(4.2 \%)$ \\
\hline
\end{tabular}

HCC, hepatocellularcarcinoma; CC, cholangiocarcinoma; Others includes neuroendocrine tumor, myofibroblastic tumor, chronic inflammation, and necrotic nodule.

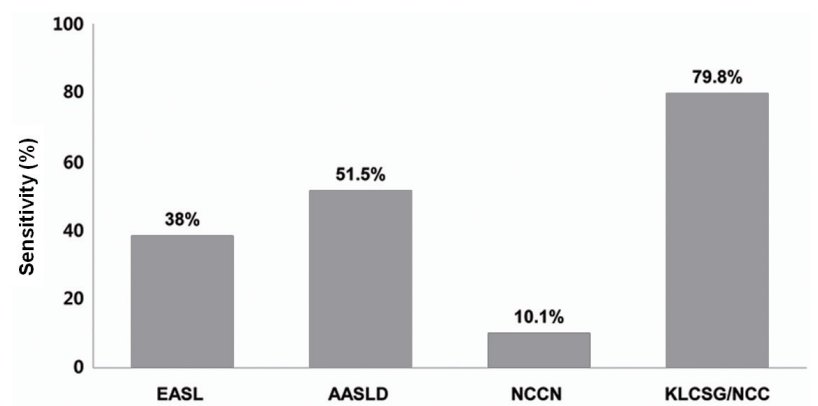

Figure 1. Comparison of sensitivity among clinical diagnostic criteria for hepatocellular carcinoma. The sensitivity was the highest using the KLCSG/NCC criteria (79.8\%), followed by the AASLD (51.5\%), EASL (38.4\%), and NCCN (10.1\%) criteria. The sensitivity differed significantly between the patient groups $(P<0.001)$.

level was below $200 \mathrm{ng} / \mathrm{mL}$ in 274 patients (77.1\%). Assessing the tumor size by the largest diameter on imaging studies, 62 patients (17.5\%) revealed tumor size $<2 \mathrm{~cm}$ and $293(82.5 \%)$ had tumor size $\geq 2 \mathrm{~cm}$. Two hundred and sixty-four patients $(74.4 \%)$ received hepatic resection, four (1.1\%) underwent liver transplantation, 72 (20.3\%) went through liver biopsy, and 15 (4.2\%) received both liver biopsy and hepatic resection. As for histologic diagnosis, HCC was found in 284 patients (80.0\%), followed by cholangiocarcinoma (CC) in $23(6.5 \%)$, HCC-CC in $13(3.7 \%)$, and focal nodular hyperplasia in $10(2.8 \%)$ (Table 3$)$.

\section{Comparison of accuracy among clinical diag- nostic criteria in total patients}

In a total of 355 patients, sensitivity was highest in KLCSG/NCC criteria (79.8\%), followed by AASLD criteria (51.5\%), EASL criteria (38.4\%), NCCN criteria (10.1\%) (Fig. 1). The difference of sensitivities among the criteria were statistically significant $(P<0.001)$.

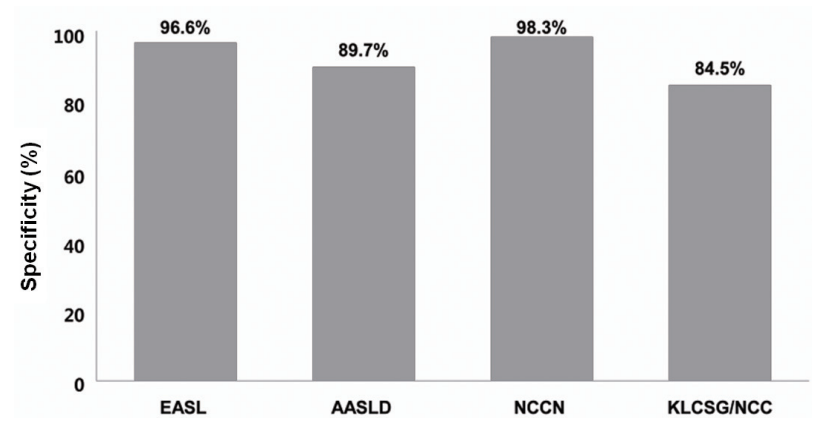

Figure 2. Comparison of specificity among the clinical diagnostic criteria for hepatocellular carcinoma. The specificity was $96.6 \%$ for EASL, $89.7 \%$ for AASLD, $98.3 \%$ for NCCN, and $84.5 \%$ for KLSCG/NCC criteria. The differences between the criteria were not statistically significant $(P=0.05)$.

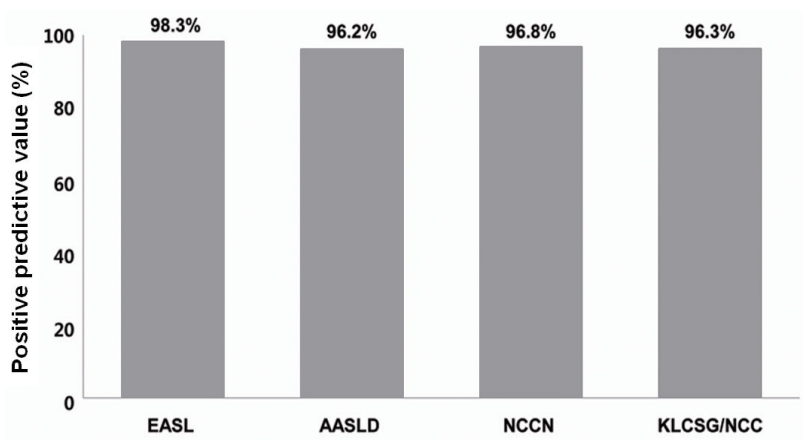

Figure 3. Comparison of positive predictive values (PPVs) among the clinical diagnostic criteria for hepatocellular carcinoma. The PPV was similar among the four noninvasive criteria $(98.3 \%, 96.2 \%, 96.8 \%$, and 96.3\% for EASL, AASLD, NCCN, and KLSCG/NCC criteria, respectively).

Specificity was not significantly different among the four criteria $(96.6 \%, 89.7 \%, 84.5 \%$, and $98.3 \%$, for EASL, AASLD, KLSCGNCC, and NCCN criteria, respectively, $P=0.05$ ) (Fig. 2). PPV was also similar among the four non-invasive criteria (Fig. 3).

\section{Accuracy of clinical diagnostic criteria in $\mathrm{HBsAg}$ positive and negative patients}

Histologically, HCC was confirmed in 234 (92.5\%) of $253 \mathrm{HBsAg}$ positive patients (HBsAg (+) group) and in $63(61.8 \%)$ of $102 \mathrm{HB}$ sAg (-) patients (HBsAg (-) group) $(P<0.001)$. While the sensitivity of EASL, AASLD, and KLCSG/NCC criteria in the HBsAg (+) group was significantly higher than in the $\mathrm{HBsAg}(-)$ group $(42.7 \%$ vs. $22.2 \%, P=0.03$ for EASL; $59.0 \%$ vs. $23.8 \%, P<0.001$ for AASLD, $91.0 \%$ vs. $38.1 \%, P<0.001$ for KLCSG/NCC criteria), the sensitivity of the NCCN criteria was similar between the two groups. Specificity of KLCSG/NCC in HBsAg (+) group was significantly lower than in the HBsAg (-) group (63.2\% vs. $94.9 \%, P=0.03)$. 
Table 4. Accuracy of the clinical diagnostic criteria in HBsAg (+) and HBsAg (-) patients

\begin{tabular}{|c|c|c|c|}
\hline & HBsAg (+) & HBsAg (-) & $P$-value \\
\hline Histologically confirmed HCC & 234/253 (92.5\%) & 63/102 (61.8\%) & $<0.001$ \\
\hline \multicolumn{4}{|l|}{ EASL } \\
\hline Sensitivity & 100/234 (42.7\%) & 14/63 (22.2\%) & 0.03 \\
\hline Specificity & 18/19 (94.7\%) & $38 / 39$ (97.4\%) & 1.00 \\
\hline PPV & 100/101 (99.0\%) & 14/15 (93.3\%) & 1.00 \\
\hline \multicolumn{4}{|l|}{ AASLD } \\
\hline Sensitivity & 138/234 (59.0\%) & 15/63 (23.8\%) & $<0.001$ \\
\hline Specificity & 15/19 (79.0\%) & $37 / 39$ (94.9\%) & 0.75 \\
\hline PPV & 138/142 (97.2\%) & 15/17 (88.2\%) & 1.00 \\
\hline \multicolumn{4}{|l|}{ KLCSG/NCC } \\
\hline Sensitivity & 213/234 (91.0\%) & 24/63 (38.1\%) & $<0.001$ \\
\hline Specificity & $12 / 19(63.2 \%)$ & $37 / 39(94.9 \%)$ & 0.03 \\
\hline PPV & $213 / 220(96.8 \%)$ & $24 / 26(92.3 \%)$ & 1.00 \\
\hline \multicolumn{4}{|l|}{ NCCN } \\
\hline Sensitivity & 19/234 (8.1\%) & 11/63 (17.5\%) & 0.32 \\
\hline Specificity & 18/19 (94.7\%) & $39 / 39(100 \%)$ & 1.00 \\
\hline PPV & 19/20 (95.0\%) & 11/11 (100\%) & 1.00 \\
\hline
\end{tabular}

HCC, hepatocellularcarcinoma; EASL, European Association for the Study of the Liver; AASLD, the American Association for the Study of Liver Diseases; KLCSG/NCC, the Korean Liver Cancer Study Group and the National Cancer Center; NCCN, National Comprehensive Cancer Network; PPV, positive predictive value.

Specificity of other criteria was not different between the HBsAg (+) and (-) groups. PPV of all criteria showed no significant difference between the two groups (Table 4).

\section{Accuracy of clinical diagnostic criteria in pa- tients with and without LC}

Histologic examination showed HCC in 169 (93.8\%) of 180 patients with LC (LC group) and in $128(73.1 \%)$ of 175 patients without $\mathrm{LC}$ (non-LC group) $(P<0.001)$. While the sensitivity of $\mathrm{KLC}-$ SG/NCC criteria in LC group was significantly higher ( $89.9 \%$ vs. $66.4 \%, P<0.001)$, their specificity was lower $(45.5 \%$ vs. $93.6 \%$, $P<0.001)$, compared to non-LC group. PPV of KLCSG/NCC was not different between the two groups. Accuracy of the NCCN criteria was similar between the LC group and the non- $\mathrm{LC}$ group (Table 5).

\section{Accuracy of clinical diagnostic criteria accord- ing to tumor size}

Histologic examination proved HCC in 48 (77.4\%) of 62 patients with tumor size $<2 \mathrm{~cm}$ and in 249 (84.9\%) of 293 patients with a tumor sized $\geq 2 \mathrm{~cm}(P=0.18)$. The sensitivities, specificities, and PPVs of these criteria did not show any significant difference between the two groups (Table 6).

\section{Comparison of sensitivity and specificity among clinical diagnostic criteria in $\mathrm{HBsAg}(+)$ or (-) subgroups with or without LC}

As described above, sensitivity and specificity of non-invasive diagnostic criteria showed significant differences according to HBsAg and LC status. PPV was similar in all patients. In addition, no difference in diagnostic accuracy was noted according to tumor size. Hence, we compared the sensitivity and specificity of clinical diagnostic criteria in subgroups with or without $\mathrm{HBsAg}$ and/or LC.

In the HBsAg (+) LC (+) subgroup, sensitivity of AASLD and KLCSG/NCC criteria was high $(92.0 \%$ and $91.3 \%$, respectively), followed by EASL criteria (66.7\%) and NCCN criteria (6.0\%, $P<0.001)$. In HBsAg (-) LC (+) subgroup, sensitivity of EASL, AASLD, and KLCSG/NCC criteria was higher $(73.7 \%, 78.9 \%$, and 
Table 5. Accuracy of clinical diagnostic criteria in patients with or without liver cancer

\begin{tabular}{|c|c|c|c|}
\hline & $\mathrm{LC}(\mathrm{n}=180)$ & Non-LC $(n=175)$ & $P$-value \\
\hline Histologically confirmed HCC & 169/180 (93.8\%) & 128/175 (73.1\%) & $<0.001$ \\
\hline \multicolumn{4}{|l|}{ EASL } \\
\hline Sensitivity & $114 / 169$ (67.5\%) & N/A & N/A \\
\hline Specificity & 9/11 (81.8\%) & N/A & N/A \\
\hline PPV & 114/116 (98.3\%) & N/A & N/A \\
\hline \multicolumn{4}{|l|}{ AASLD } \\
\hline Sensitivity & $153 / 169(90.5 \%)$ & N/A & N/A \\
\hline Specificity & $5 / 11(45.5 \%)$ & N/A & N/A \\
\hline PPV & 153/159 (96.2\%) & N/A & N/A \\
\hline \multicolumn{4}{|l|}{ KLCSG/NCC } \\
\hline Sensitivity & 152/169 (89.9\%) & $85 / 128(66.4 \%)$ & $<0.001$ \\
\hline Specificity & $5 / 11(45.5 \%)$ & $44 / 47(93.6 \%)$ & $<0.001$ \\
\hline PPV & 152/158 (96.2\%) & $85 / 88$ (96.6\%) & 1.00 \\
\hline \multicolumn{4}{|l|}{ NCCN } \\
\hline Sensitivity & 12/169 (7.1\%) & $18 / 128(14.1 \%)$ & 0.48 \\
\hline Specificity & 11/11 (100\%) & 46/47 (97.9\%) & 1.00 \\
\hline PPV & $12 / 12(100 \%)$ & $18 / 19(94.7 \%)$ & 1.00 \\
\hline
\end{tabular}

LC, liver cirrhosis; HCC, hepatocellular carcinoma; EASL, European Association for the Study of the Liver; AASLD, the American Association for the Study of Liver Diseases; KLCSG/NCC, the Korean Liver Cancer Study Group and the National Cancer Center; NCCN, National Comprehensive Cancer Network; PPV, positive predictive value; N/A, not available.

$78.9 \%$, respectively) than $15.8 \%$ of NCCN criteria $(P=0.003)$. In the HBsAg (+) LC (-) subgroup, the KLCSG/NCC criteria still showed a high sensitivity of $90.5 \%$ (Fig. 4).

In the HBsAg (+) LC (+) subgroup, specificity of NCCN criteria was highest $(100.0 \%)$, followed by the EASL criteria (85.7\%) and the AASLD and KLCSG/NCC criteria ( $42.9 \%$ for both, $P=0.93)$. In the HBsAg (-) LC (+) subgroup, NCCN criteria showed specificity of $100 \%$, followed by EASL criteria $(75.0 \%)$ and the AASLD and KLCSG/NCC criteria (50.0\% for both, $P=1.00$ ) (Fig. 5).

\section{Usefulness of clinical diagnostic criteria based on sensitivity and false positive rate}

Usefulness of clinical diagnostic criteria was compared based on sensitivity and false positive rate (Table 7). In the whole patients, all four criteria showed acceptable FPR $(<5 \%)$ and KLCSG/NCC criteria presented the highest sensitivity $(79.8 \%)$, Subgroup analysis was done according to the presence of $\mathrm{HBsAg}, \mathrm{LC}$, and tumor size. In HBsAg (+) group, EASL, AASLD, and KLCSG/NCC criteria showed acceptable FPR and KLCSG/NCC criteria present the highest sensitivity of $91.0 \%$. In HBsAg (-) group, FPR of NCCN criteria was $<5 \%$ but its sensitivity is too low (17.5\%). In LC (+) group, all four criteria showed acceptable FPR; AASLD and KLCSG/NCC criteria presented high sensitivity of $90.5 \%$ and $89.9 \%$, respectively. In LC (-) group, KLCSC/NCC criteria showed low FPR (3.4\%) and relatively high sensitivity (66.4\%), compared to other criteria. In patients with tumor $2 \mathrm{~cm}$, all four criteria showed acceptable FPR and KLCSG/NCC criteria presented the highest sensitivity of $79.9 \%$. In patients with tumor $<2 \mathrm{~cm}$, FPR of NCCN was low but its sensitivity is too low (2.1\%).

\section{DISCUSSION}

Most guidelines recommend non-invasive criteria for diagnosing HCC. Their usefulness was not fully verified in HBV endemic areas. Moreover, there have been no attempts to compare the accuracy of these guidelines until now. Hence, we compared the usefulness of non-invasive diagnostic criteria by four representative guidelines for management of HCC by EASL, AASLD, KLCSG/NCC, and NCCN. We further analyzed their accuracy according to HBsAg status, accompanying $\mathrm{LC}$, and tumor size in a HBV endemic area.

Our study revealed that sensitivity was the highest in KLCSG/ NCC criteria (79.8\%), followed by AASLD (51.5\%), EASL (38.4\%), and NCCN criteria $(10.1 \%, P<0.001)$, whereas specificity (84.598.3\%) and PPV (96.2-98.3\%) were quite similar in the whole patients. Subgroup analysis showed that EASL and AASLD criteria were more sensitive $(42.7 \%$ vs. $22.2 \%, P=0.03$ for EASL; $59.0 \%$ vs. $23.8 \%, P<0.001$ for AASLD) and similarly specific in HBsAg (+) patients than in HBsAg (-) patients. KLCSG/NCC criteria showed 
Table 6. Accuracy of clinical diagnostic criteria according to tumor size

\begin{tabular}{|c|c|c|c|}
\hline & $<2 \mathrm{~cm}$ & $\geq 2 \mathrm{~cm}$ & $P$-value \\
\hline Histologically confirmed HCC & 48/62 (77.4\%) & 249/293 (84.9\%) & 0.18 \\
\hline \multicolumn{4}{|l|}{ EASL } \\
\hline Sensitivity & N/A & $114 / 249(45.8 \%)$ & N/A \\
\hline Specificity & N/A & $42 / 44(95.5 \%)$ & N/A \\
\hline PPV & N/A & $114 / 116(98.3 \%)$ & N/A \\
\hline \multicolumn{4}{|l|}{ AASLD } \\
\hline Sensitivity & $29 / 48(60.4 \%)$ & $124 / 249(49.8 \%)$ & 1.00 \\
\hline Specificity & $12 / 14(85.7 \%)$ & 40/44 (90.9\%) & 1.00 \\
\hline PPV & 29/31 (93.5\%) & 124/128 (96.9\%) & 1.00 \\
\hline \multicolumn{4}{|l|}{ KLCSG/NCC } \\
\hline Sensitivity & $38 / 48(79.2 \%)$ & 199/249 (79.9\%) & 1.00 \\
\hline Specificity & $11 / 14(78.6 \%)$ & $38 / 44(86.4 \%)$ & 1.00 \\
\hline PPV & $38 / 41(92.7 \%)$ & 199/205 (97.1\%) & 1.00 \\
\hline \multicolumn{4}{|l|}{ NCCN } \\
\hline Sensitivity & $1 / 48(2.1 \%)$ & 29/249 (11.7\%) & 0.56 \\
\hline Specificity & 14/14 (100\%) & $43 / 44$ (97.7\%) & 1.00 \\
\hline PPV & $1 / 1(100 \%)$ & 29/30 (96.7\%) & 1.00 \\
\hline
\end{tabular}

HCC, hepatocellular carcinoma; EASL, European Association for the Study of the Liver; AASLD, the American Association for the Study of Liver Diseases; KLCSG/NCC, the Korean Liver Cancer Study Group and the National Cancer Center; NCCN, National Comprehensive Cancer Network; PPV, positive predictive value; N/A, not available.

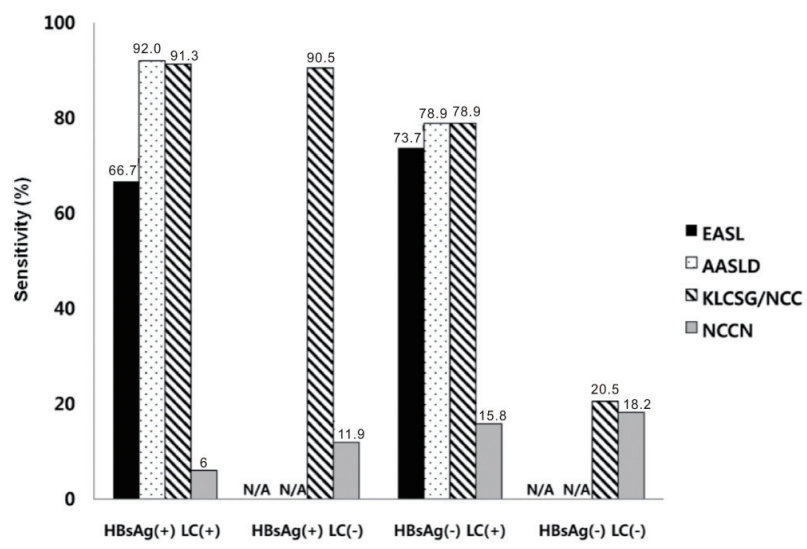

Figure 4. Comparison of sensitivity among the clinical diagnostic criteria in $\mathrm{HBsAg}(+)$ and $\mathrm{HBsAg}(-)$ subgroups with or without liver cancer (LC). The sensitivities of the AASLD and KLCSG/NCC criteria were highest in the HBsAg (+) LC (+) subgroup (92.0\% and 91.3\%, respectively), followed by the EASL criteria $(66.7 \%, P<0.001)$. The sensitivities of the EASL, AASLD, and KLCSG/NCC criteria in the HBsAg (-) LC (+) subgroup were $73.7 \%, 78.9 \%$, and $78.9 \%$, respectively. It is noteworthy that the KLCSG/NCC criteria had a sensitivity of $90.5 \%$ in the HBsAg (+) LC (-) subgroup.

higher sensitivity $(91.0 \%$ vs. $38.1 \%, P<0.001)$ but lower specificity (63.2\% vs. $94.9 \%, P=0.03$ ) in patients with HBV infection, com-

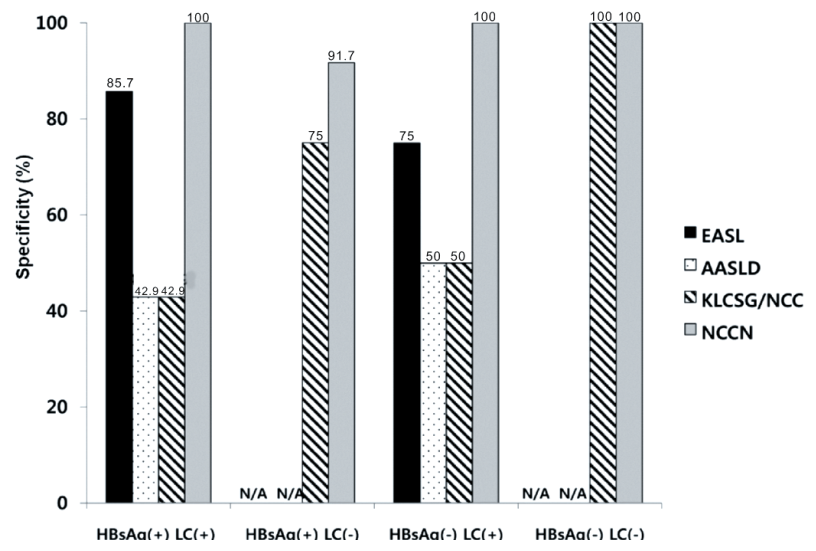

Figure 5. Comparison of specificity among the clinical diagnostic criteria in the HBsAg (+) and HBsAg (-) subgroups with or without LC. In the LC (+) subgroup with or without HBV infection, the NCCN criteria had a specificity of $100 \%$; the specificity of the EASL criteria was $85.7 \%$ for the HBsAg (+) group and $75.0 \%$ for the HBsAg (-) group.

pared to those without. Similarly, sensitivity of KLCSG/NCC was higher (89.9\% vs. $66.4 \%, P<0.001)$ but its specificity was lower ( $45.5 \%$ vs. $93.6 \%, P<0.001)$ in LC group than non-LC group. Notably, its sensitivity was $90.5 \%$ and specificity was $75 \%$ in $\mathrm{HBsAg}$ (+) patients without LC. NCCN criteria showed high specificity but 
Table 7. Sensitivity and false-positive rate (FPR) of the clinical diagnostic criteria

\begin{tabular}{|c|c|c|c|c|}
\hline Criteria & EASL & AASLD & NCCN & KLCSG/NCC \\
\hline (\%) & Sensitivity/FPR & Sensitivity/FPR & Sensitivity/FPR & Sensitivity/FPR \\
\hline Overall & $38.4 / 1.7$ & $51.5 / 3.8$ & $10.1 / 3.2$ & $79.8 / 3.7$ \\
\hline HBsAg (+) & $42.7 / 1$ & $59.0 / 2.8$ & $8.1 / 5$ & $91.0 / 3.2$ \\
\hline $\operatorname{HBsAg}(-)$ & $22.2 / 6.7$ & 23.8/11.8 & $17.5 / 0$ & $38.1 / 7.7$ \\
\hline LC (+) & $67.5 / 1.7$ & $90.5 / 3.8$ & $7.1 / 0$ & $89.9 / 3.8$ \\
\hline LC $(-)$ & NA/NA & NA/NA & $14.1 / 5.3$ & $66.4 / 3.4$ \\
\hline Tumor size $\geq 2 \mathrm{~cm}$ & $45.8 / 1.7$ & $49.8 / 3.1$ & $11.7 / 3.3$ & $79.9 / 2.9$ \\
\hline Tumor size <2cm & NA/NA & $60.4 / 6.5$ & $2.1 / 0$ & $79.2 / 7.3$ \\
\hline
\end{tabular}

"The measure of all the values is "\%".

EASL, European Association for the Study of the Liver; AASLD, the American Association for the Study of Liver Diseases; KLCSG/NCC, the Korean Liver Cancer Study Group and the National Cancer Center; NCCN, National Comprehensive Cancer Network; FPR, false positive rate.

very low sensitivity throughout the subgroups.

For all evaluable diagnostic criteria, accuracy was not different according to tumor size.

Three published studies explored the accuracy of non-invasive diagnostic criteria for HCC by comparing pathologic and clinical diagnosis. ${ }^{12,18,19} \mathrm{Kim}$ et al ${ }^{12}$ validated AASLD criteria in 206 patients with liver nodules larger than $2 \mathrm{~cm}$. According to this report, diagnosis by AASLD criteria revealed that sensitivity, specificity, and PPV were $89.2 \%, 76.2 \%$, and $93.6 \%$.

Park et a ${ }^{18}$ evaluated the accuracy of KLCSG/NCC criteria for clinical diagnosis of HCC in 232 patients with liver nodules of variable sizes ( $(\mathrm{C}=49.1 \%$, HBV infection $=73.3 \%)$. They reported that overall sensitivity, specificity, and PPV were $95.1 \%, 73.9 \%$, and $93.7 \%$, repectively. The accuracy was not significantly affected by lesion size or the presence of clinical cirrhosis. ${ }^{18}$ Forner et al19performed a prospective validation of the AASLD criteria in the diagnosis of 89 hepatic nodules $\leq 20 \mathrm{~mm}$ in cirrhotics (HCV infection $=76.4 \%$ ). 19 They reported that sensitivity, specificity, and PPV were $33 \%, 100 \%$, and $100 \%$, repectively.

In addition, two other studies are noteworthy. Leoni et a ${ }^{20}$ evaluated 75 liver nodules in patients with LC (HCV infection $=55 \%$, size $=10-30 \mathrm{~mm}$ ). However, biopsy was done only if clinical diagnostic criteria were not fulfilled. Sangiovanni et a ${ }^{21}$ explored de novo 67 liver nodules in LC patients (HCV infection $=62 \%$ ). Although histologic findings were available in all patients, fulfillment of the clinical criteria was not evaluated. Hence, accuracy of the non-invasive criteria could not be gained in the previous two studies. $^{20,21}$

While HCC was confirmed in $284(80.0 \%)$ of 355 patients enrolled in this study, final histologic diagnosis were CC in $23(6.5 \%)$, HCC-CC in $13(3.7 \%)$, focal nodular hyperplasia in $10(2.8 \%)$, eosinophilic abscess, abscess, and regenerating nodule. Forner et $\mathrm{al}^{19}$ reported that $67.4 \%$ of patients with liver nodule $\leq 20 \mathrm{~mm}$ detected during ultrasound surveillance were proven to be HCC, whereas others were diagnosed with CC $(1.1 \%)$, regenerative nodules/dysplastic nodules (27.0\%), hemangioma (3.4\%), and focal nodular hyperplasia (1.1\%). According to Sangiovanni et al ${ }^{21}$ among 67 liver nodules (1-2 cm in size), 44 (66\%) nodules were finally confirmed as HCC, two (3\%) as CC, three (4\%) as low grade dysplastic nodules, and $18(27 \%)$ as macroregenerative nodule. ${ }^{21}$ According to Park et al ${ }^{18} 12$ of 189 patients (6.3\%) were falsely diagnosed as HCC using KLCSG/NCC criteria; six cases of CC, two of dysplastic nodules, one of focal nodular hyperplasia, two of hamartoma, and one of angiomyolipoma. ${ }^{18}$

Among all enrolled patients in this study, KLCSG/NCC criteria showed the highest sensitivity, followed by AASLD, EASL, and NCCN criteria, whereas the specificity of these criteria were quite high and not significantly different between these criteria. The EASL and AASLD criteria were more sensitive and similarly specific in HBsAg (+) patients than in HBsAg (-) patients. KLCSG/NCC criteria showed high sensitivity but low specificity in patients with HBV infection or LC. Different sensitivity and specificity among these criteria according to $\mathrm{HBsAg}$ status and presence of $\mathrm{LC}$ can be explained by disagreement on detailed clinical definition of HCC among the guidelines as well as unique characteristics of patients included in this study ( $L C=50.7 \%$, HBV infection=71.3\%). EASL, AASLD, and KLCSG/NCC guideline shares common elements for non-invasive diagnostic criteria of HCC but their detailed definition was quite different among the guidelines (Table 1). First of all, patients with $L C$ are defined as the only risk group in EASL and AASLD criteria, whereas those with HBV and HCV infections are also regarded as a risk group in the KLCSG/NCC criteria. Actually, 
$84(87.5 \%)$ of $96 \mathrm{HBsAg}(+)$ patients without LC in this study were proven to have HCC, which led to increased sensitivity of KLCSG/ NCC criteria. In addition, the cut-off level of serum AFP and image findings required for non-invasive diagnosis are most strict in EASL criteria (except NCCN criteria), followed by the AASLD and KLCSG/ NCC criteria. To a certain extent, tendency toward high sensitivity and low specificity of KLCSG/NCC and AASLD criteria might be explained by relatively loose standard for serum AFP level and image findings.

KLCSG/NCC criteria showed higher sensitivity $(91.0 \%$ vs. $38.1 \%, P<0.001)$ but lower specificity $(63.2 \%$ vs. $94.9 \%, P=0.03)$ in patients with $\mathrm{HBV}$ infection, compared to those without. These findings are constant with the previous reports showing that its sensitivity were $97.3 \%$ and $86.8 \%$ in the HBsAg potisitive group and non-HBV group, respectively $(P<0.001)$, and the specificity were $56.5 \%$ and $91.3 \%$, respectively $(P<0.001) .{ }^{19}$ Remarkably, KLCSG/NCC criteria showed high sensitivity in the HBsAg (+) group regardless of combined LC (91.3\% with LC vs. $90.5 \%$ without LC). Furthermore, specificity of KLCSG/NCCN criteria in HBsAg (+) LC (-) group is quite acceptable (75.0\%) in this study. Hence, it should be positively considered to include HBV-infected patients in risk group for clinical diagnostic criteria of HCC irrespective of LC status in HBV endemic areas.

Expanded risk group and inclusion of small tumors in KLCSG/ NCC criteria can explain its high sensitivity in the overall patients and high FPR in HBsAg (-) patients and those with tumor $<2 \mathrm{~cm}$. KLCSG/NCC criteria should be applied with caution in those subgroups. In addition, further studies are warranted for evaluation of its accuracy in patient with tumor $<1 \mathrm{~cm}$.

To the best of our knowledge, this is the first study comparing the usefulness of non-invasive diagnostic criteria by the representative guidelines in a HBV endemic area. We showed high sensitivity of KLCSG/NCC and AASLD criteria, compared to EASL criteria, and excellent specificity of all criteria. While EASL and AASLD criteria were more sensitive and similarly specific in $\mathrm{HBSAg}(+)$ patients, KLCSG/NCC criteria showed higher sensitivity but lower sensitivity in those with HBV infection or LC. Notably, the KLCSG/ NCC criteria showed high sensitivity and acceptable specificity in HBsAg (+) patients without LC. When we compared the usefulness of clinical diagnostic criteria based on sensitivity and FPR, KLCSG/ NCC criteria showed acceptable FPR and the highest sensitivity in the overall patients, HBsAg (+) group, LC (-) group, and patients with tumor $\geq 2 \mathrm{~cm}$. Its sensitivity is similar to that of AASLD in LC (+) group (89.9\% vs. $90.5 \%)$. Hence, inclusion of HBV infection in a risk group of clinical diagnostic criteria for HCC would be reason- able to improve sensitivity with acceptable FPR in HBV endemic areas.

We acknowledge that our study had some limitations as a retrospective study. To a certain degree, our data might be affected by selection bias and personal preference of physicians, even though a concerted effort had been put into keeping consistent diagnostic and therapeutic strategy in our institution. Patients with typical image findings and elevated serum AFP who did not underwent surgery were most likely to be excluded in this study since biopsy were rarely performed for those patients. In addition, patients with multiple tumors were excluded in this study since we can not identify which tumor contributes to elevation of serum AFP level. Despite a few limitations, we investigated a considerable number of patients recruited from a single institution during recent two years, which gives a homogeneous character to this study. Standardized up-to-date techniques for measurement of serum AFP and dynamic imaging ${ }^{22}$ and coherent strategy to make decision were adopted for the enrolled patients.

In conclusion, overall sensitivity was high with KLCSG/NCC and AASLD criteria, specificity was excellent with all criteria. Based on sensitivity and FPR, KLCSG/NCC criteria was the most useful one in the overall patients; especially in HBsAg (+) group, LC (-) group, and patients with tumor $\geq 2 \mathrm{~cm}$. Inclusion of HBV infection as a risk factor in clinical diagnostic criteria for HCC would be reasonable to improve sensitivity with acceptable FPR in HBV endemic areas.

\section{Conflicts of Interest}

The authors have no conflicts to disclose.

\section{REFERENCES}

1. Parkin DM, Bray F, Ferlay J, Pisani P. Estimating the world cancer burden: Globocan 2000. Int J Cancer 2001;94:153-156.

2. Perz JF, Armstrong GL, Farrington LA, Hutin YJ, Bell BP. The contributions of hepatitis $B$ virus and hepatitis $C$ virus infections to cirrhosis and primary liver cancer worldwide. J Hepatol 2006;45:529538.

3. Song IH, Kim KS. Current status of liver diseases in Korea: hepatocellular carcinoma. Korean J Hepatol 2009;15(Suppl 6):S50-S59.

4. Torzilli G, Minagawa M, Takayama T, Inoue K, Hui AM, Kubota K, et al. Accurate preoperative evaluation of liver mass lesions without fine-needle biopsy. Hepatology 1999;30:889-893.

5. Levy I, Greig PD, Gallinger S, Langer B, Sherman M. Resection of hepatocellular carcinoma without preoperative tumor biopsy. Ann 
Surg 2001;234:206-209.

6. Wu JT. Serum alpha-fetoprotein and its lectin reactivity in liver diseases: a review. Ann Clin Lab Sci 1990;20:98-105.

7. Ayyappan AP, Jhaveri KS. CT and MRI of hepatocellular carcinoma: an update. Expert Rev Anticancer Ther 2010;10:507-519.

8. Colli A, Fraquelli M, Casazza G, Massironi S, Colucci A, Conte D, et al. Accuracy of ultrasonography, spiral CT, magnetic resonance, and alpha-fetoprotein in diagnosing hepatocellular carcinoma: a systematic review. Am J Gastroenterol 2006;101:513-523.

9. Durand F, Regimbeau JM, Belghiti J, Sauvanet A, Vilgrain V, Terris B, et al. Assessment of the benefits and risks of percutaneous biopsy before surgical resection of hepatocellular carcinoma. J Hepatol 2001;35:254-258

10. Bruix J, Sherman M, Llovet JM, Beaugrand M, Lencioni R, Burroughs AK, et al. Clinical management of hepatocellular carcinoma. Conclusions of the Barcelona-2000 EASL conference. European Association for the Study of the Liver. J Hepatol 2001;35:421-430.

11. Bruix J, Sherman M; Practice Guidelines Committee, American Association for the Study of Liver Diseases. Management of hepatocellular carcinoma. Hepatology 2005;42:1208-1236.

12. Kim SE, Lee HC, Shim JH, Park HJ, Kim KM, Kim PN, et al. Noninvasive diagnostic criteria for hepatocellular carcinoma in hepatic masses $>2 \mathrm{~cm}$ in a hepatitis B virus-endemic area. Liver Int 2011;31:1468-1476.

13. Benson AB 3rd, Abrams TA, Ben-Josef E, Bloomston PM, Botha $J F$, Clary BM, et al. NCCN clinical practice guidelines in oncology: hepatobiliary cancers. J Natl Compr Canc Netw 2009;7:350-391.

14. Park JW, Choi JY, Seo KS. 2009 Hepatocellular carcinoma practice guideline. Seoul: the Korean Liver Cancer Study Group and the National Cancer Center, 2009;1-56.

15. Kim SH, Lee WJ, Lim HK, Park CK. SPIO-enhanced MRI findings of well-differentiated hepatocellular carcinomas: correlation with
MDCT findings. Korean J Radiol 2009;10:112-120.

16. Lee S, Kim SH, Park CK, Kim YS, Lee WJ, Lim HK. Comparison between areas with Gd-EOB-DTPA uptake and without in hepatocellular carcinomas on Gd-EOB-DTPA-enhanced hepatobiliaryphase MR imaging: pathological correlation. J Magn Reson Imaging 2010;32:719-725.

17. Chang S, Kim SH, Lim HK, Lee WJ, Choi D, Lim JH. Needle tract implantation after sonographically guided percutaneous biopsy of hepatocellular carcinoma: evaluation of doubling time, frequency, and features on CT. AJR Am J Roentgenol 2005;185:400-405.

18. Park JW, An M, Choi Jl, Kim Yl, Kim SH, Lee WJ, et al. Accuracy of clinical criteria for the diagnosis of hepatocellular carcinoma without biopsy in a Hepatitis B virus-endemic area. J Cancer Res Clin Oncol 2007;133:937-943.

19. Forner A, Vilana R, Ayuso C, Bianchi L, Solé M, Ayuso JR, et al. Diagnosis of hepatic nodules $20 \mathrm{~mm}$ or smaller in cirrhosis: Prospective validation of the noninvasive diagnostic criteria for hepatocellular carcinoma. Hepatology 2008;47:97-104.

20. Leoni S, Piscaglia F, Golfieri R, Camaggi V, Vidili G, Pini P, et al. The impact of vascular and nonvascular findings on the noninvasive diagnosis of small hepatocellular carcinoma based on the EASL and AASLD criteria. Am J Gastroenterol 2010;105:599-609.

21. Sangiovanni $A$, Manini MA, lavarone $M$, Romeo $R$, Forzenigo LV, Fraquelli $M$, et al. The diagnostic and economic impact of contrast imaging techniques in the diagnosis of small hepatocellular carcinoma in cirrhosis. Gut 2010;59:638-644.

22. Okada M, Imai Y, Kim T, Kogita S, Takamura M, Kumano S, et al. Comparison of enhancement patterns of histologically confirmed hepatocellular carcinoma between gadoxetate- and ferucarbotranenhanced magnetic resonance imaging. J Magn Reson Imaging 2010;32:903-913. 